\begin{tabular}{|l|l|}
\hline $\begin{array}{l}\text { Instituto de } \\
\text { Geriatria e Gerontologia }\end{array}$ & $\begin{array}{l}\text { Pan American Journal of Aging Research } \\
\text { PAJAR, Porto Alegre, v. 8, p. 1-8, jan.-dez. } 2020 \\
\text { ISSN-L: 2357-9641 }\end{array}$ \\
\hline http://dx.doi.org/10.15448/2357-9641.2020.1.37707 & \\
\cline { 2 - 3 }
\end{tabular}

\title{
Avaliação do paladar de idosos e sua relação com estado nutricional e hábitos alimentares
}

\author{
Evaluation of the taste of older people and their relationship with nutritional state and \\ food habits
}

Evaluación del sabor de las personas mayores y su relación con el estado nutricional y los hábitos alimentarios

\section{Darlise Rodrigues dos \\ Passos Gomes ${ }^{1}$ \\ orcid.org/0000-0002-6534-2795 \\ darlise.passos@gmail.com}

\section{Claudine Lamanna}

\section{Schirmer ${ }^{2}$}

orcid.org/0000-0003-3803-740X nutricionistaclaudine@gmail.com

\section{Ângela de Oliveira Antunez Nolte ${ }^{2}$}

orcid.org/0000-0002-7406-570X aoanolte@gmail.com

\section{Ângelo José Gonçalves \\ Bós ${ }^{2}$ \\ orcid.org/0000-0003-4901-3155 angelo.bos@pucrs.br}

\section{Janaína Guimarães Venzke 3 \\ orcid.org/0000-0003-3990-3912 janaina.venzke@ufrgs.br}

Recebido em: 15/4/2020. Aprovado em: 31/8/2020. Publicado em: 13/11/2020.

\section{(c) (1)}

Artigo está licenciado sob forma de uma licença Creative Commons Atribuição 4.0 Internacional.

\section{Resumo}

Objetivos: avaliar a prevalência de disgeusia (distorção ou diminuição do paladar) e a associação com o estado nutricional e as práticas alimentares em um grupo de idosos, assim como identificar os gostos mais afetados e preservados. Métodos: estudo transversal com amostra recrutada no Centro de Lazer para idosos no municipio de Porto Alegre, RS. Avaliou-se o paladar, através de tiras gustativas ("tastestrips"), e o estado nutricional, através do Índice de Massa Corporal. As práticas alimentares foram investigadas através de questionário. Para análise estatística utilizou-se os testes qui-quadrado e ANOVA, com nivel de significância de $p<0,05$ Resultados: foram avaliados 62 idosos ( $84 \%$ feminino, média de $70 \pm 7,6$ anos), prevalência de disgeusia foi $19,4 \%(n=12)$, detectou-se $58 \%$ de sobrepeso $(n=36)$, $40,5 \%$ de eutrofia ( $n=25)$ e $1,5 \%$ de baixo peso $(n=1)$, não sendo encontrada associação entre alteração de paladar e estado nutricional $(p=0,397)$. Cerca de $55 \%$ dos idosos apresentaram consumo elevado de sódio. Não houve diferença na média de pontos para o gosto ácido entre os idosos que temperam a salada com limão ou vinagre e os que não o fazem ( $p=0,054$ e $p=0,935$, respectivamente). A média de pontos para o gosto salgado não diferiu entre os que preparam as refeições com temperos ricos em sódio e os que não os utilizam $(p=0,055)$. 0 doce foi o gosto mais preservado, enquanto o ácido mostrou-se mais reduzido. Conclusões: apesar de evidências contrárias, não se identificou impacto das alterações de paladar sobre o estado nutricional e as práticas alimentares. Ainda assim, novos estudos que incluam essas variáveis se fazem necessários.

Palavras-chave: paladar, estado nutricional, hábitos alimentares, idoso, disgeusia.

\section{Abstract}

Aims: to assess the prevalence of dysgeusia (reduced taste) and the association with nutritional status and eating practices in a group of elderly people, as well as to identify the most affected and preserved tastes.

Methods: cross-sectional study with a sample recruited at Leisure Center for the elderly in the city of Porto Alegre / RS. Taste was assessed using taste strips and nutritional status using the Body Mass Index. Eating practices were investigated through a questionnaire. For statistical analysis, the chi-square and ANOVA tests were used, with a significance level of $p<0.05$

Results: 62 elderly people ( $84 \%$ female, mean $70 \pm 7.6$ years) were evaluated, prevalence of dysgeusia was $19.4 \%(n=12), 58 \%$ overweight $(n=36), 40.5 \%$ eutrophy $(n=25)$ and $1.5 \%$ of low weight $(n=1)$, with no significant association between changes in taste and nutritional status $(p=0.397)$. Around $55 \%$ of the elderly had elevated sodium consumption. There was no significant difference in the mean of points for acid taste between the elderly who season the salad with lemon or vinegar and those who do not $(p=0.054$ and $p=0.935$, respectively). The average points for salty taste did not differ between those who prepare meals with spices rich in sodium and those who do not use them $(p=0.055)$. The sweet was the 
most preserved taste, while the acid was more reduced. Conclusions: despite the contrary evidence, the impact of taste changes on nutritional status and eating practices was not identified. Even so, new studies that include these variables are necessary.

Keywords: taste, nutritional status, food habits, aged, dysgeusia.

\section{Resumen}

Objetivos: evaluar la prevalencia de disgeusia (decremento del gusto) y la asociación con el estado nutricional y los hábitos alimentarios en un grupo de personas ancianas, asi como identificar los gustos más afectados y conservados.

Métodos: estudio transversal con una muestra reclutada en el Centro de ocio para personas mayores en la ciudad de Porto Alegre / RS. El sabor se evaluó utilizando tiras gustativas y el estado nutricional utilizando el indice de masa corporal. Las prácticas alimentarias se investigaron mediante un cuestionario. Para el análisis estadístico, se utilizaron las pruebas de chi-cuadrado y ANOVA, con un nivel de significancia de $\mathrm{p}<0.05$.

Resultados: 62 personas de edad avanzada (84\% mujeres, promedio de $70 \pm 7.6$ años) fueron evaluadas, la prevalencia de disgeusia fue de $19.4 \%(n=12), 58 \%$ de sobrepeso $(n=36)$, se detectaron $40.5 \%$ de peso normal $(n=25)$ y $1.5 \%$ de bajo peso $(n=1)$, sin asociación significativa entre los cambios en el sabor y el estado nutricional $(p=0.397)$. Alrededor del $55 \%$ de los ancianos tenían un alto consumo de sodio. No hubo diferencias significativas en la media de los puntos para el sabor ácido entre los ancianos que sazonan la ensalada con limón o vinagre y aquellos que no $(p=0.054$ y $p=0.935$, respectivamente). Los puntos promedio para el sabor salado no difirieron entre los que preparan comidas con especias ricas en sodio y los que no los usan ( $p=$ 0.055). El dulce fue el sabor más conservado, mientras que el ácido se redujo más.

Conclusiones: a pesar de la evidencia contraria, no se identificó el impacto de los cambios de sabor en el estado nutricional y las prácticas alimentarias. Aun así, son necesarios nuevos estudios que incluyan estas variables. Palabras clave: gusto, estado nutricional, hábitos alimentarios, anciano, disgeusia.

\section{Introdução}

Durante o envelhecimento ocorrem mudanças anatômicas e fisiológicas nos sistemas sensoriais - gustativo e olfativo -, produzindo resultados conflitantes na percepção do sabor dos alimentos. ${ }^{1,2,3}$ Inúmeros estudos confirmam uma diminuição gustativa associada à idade, demonstrando que os idosos são menos sensiveis para todos os gostos quando comparados aos jovens. ${ }^{4,5,6,7.8}$

As disfunções sensoriais tendem a começar ao redor dos 60 anos de idade e tornam-se mais graves após os 70 anos. ${ }^{9}$ Elas são comumente negligenciadas por não serem consideradas situações críticas, entretanto, possuem importância não apenas em termos de preferências alimentares e do prazer relacionado à comida, mas também por poderem resultar em perda de peso, prejuizo na imunidade e deterioração das condições de saúde.4,10

Diversas condições podem contribuir na etiologia das alterações gustativas, ${ }^{11}$ entre elas: presença de doenças e uso de medicamentos específicos, tratamento quimio - e radioterápico -, fumo, ${ }^{12}$ má higiene bucal, uso de próteses dentárias, ${ }^{13}$ perda de dentição, ${ }^{8}$ além da diminuição no número de corpúsculos gustativos. Os distúrbios do paladar são classificados em perda parcial (hipogeusia), perda total (ageusia) e qualquer sensação de alteração (disgeusia), sendo que a hipogeusia no idoso tem sua severidade e duração dependente do estado geral de saúde e atinge áreas de receptores gustativos que geralmente comprometem todo o espectro de gostos: ácido, amargo, doce e salgado. ${ }^{8}$

Os receptores do paladar participam sensorialmente do monitoramento da ingestão de alimento, juntamente com o olfato, o tato e a temperatura, regulando o comportamento e a homeostase corporal. Assim, a deficiência no paladar para identificar substâncias pode estar associada ao desenvolvimento de desordens que incluem obesidade, hipertensão, má nutrição e diabetes. ${ }^{14}$ Estudos $^{15}$ salientam, ainda, que a alteração da percepção sensorial é uma das disfunções fisiológicas que interferem de maneira mais importante na ingestão alimentar e, por consequência, no estado nutricional dos idosos.

Face ao exposto, a avaliação da sensibilidade gustativa se faz necessária na prática clínica. ${ }^{6}$ Entretanto, a prevalência de disgeusia em idosos, assim como a associação com parâmetros nutricionais, ainda hoje, não se encontram bem estabelecidas na literatura. Nesse sentido, o presente estudo avaliou a prevalência de disgeusia em uma amostra de idosos, identificando os sabores mais afetados e mais preservados e, buscou verificar possivel associação com o estado nutricional e as práticas alimentares.

\section{Métodos}

Os idosos foram recrutados junto ao Centro de Lazer e Recreação do Idoso (CELARI), per- 
tencente à Universidade Federal do Rio Grande do Sul (UFRGS), localizado no municipio de Porto Alegre, RS. O tamanho da amostra seguiu as recomendações minimas apresentadas em estudos envolvendo análise sensorial. ${ }^{16}$ Para as análises realizou-se um levantamento quantitativo dos que se voluntariaram a participar do estudo, e foram incluidas apenas pessoas com idade maior ou igual a 60 anos, de ambos os sexos. O estudo foi aprovado pelo Comitê de Ética em Pesquisa da UFRGS (protocolo n. ${ }^{\circ}$ 18127).

Para avaliar a função gustativa, utilizou-se o teste validado, "taste strips" 3 , o qual avalia os quatro gostos primários (doce, salgado, ácido e amargo) e consiste, basicamente, em posicionar fitas de papel filtro impregnadas com os diferentes gostos sobre a língua do individuo. Os idosos foram orientados a passar as fitas em todas as regiões da língua, sem limite de tempo, a fim de não prejudicar a identificação do gosto.

No total, foram oferecidas 18 fitas para cada participante: cada um dos gostos foi apresentado em quatro concentrações diferentes (quatro gostos vs. quatro concentrações) e mais duas fitas que não continham gosto. As substâncias utilizadas obedeceram às seguintes concentrações: sacarose 0,4; 0,2; 0,1; 0,05g/mL (doce); cloreto de sódio 0,25; 0,1; 0,$04 ; 0,016 \mathrm{~g} / \mathrm{mL}$ (salgado); cloridrato de quinino 0,006; 0,0024; 0,0009; 0,0004g/ml (amargo); e ácido cítrico 0,3; 0,165; 0,09; 0,05g/mL (ácido).

A ordem de apresentação das fitas foi sorteada para cada individuo ("pseudorandomização") a partir de um estudo validado, ${ }^{3}$ havendo 4 possibilidades diferentes para aplicação do teste. A administração das fitas respeitou a ordem crescente das concentrações dos gostos. Entre a administração das fitas, os indivíduos eram orientados a enxaguar a boca com água mineral, a fim de evitar gosto residual que pudesse fazê-los confundir os gostos seguintes.

O participante deveria escolher, a cada fita testada, uma das cinco opções: 1) doce; 2) salgado; 3) amargo; 4) ácido; 5) sem gosto. A cada fita identificada corretamente, o participante somava um ponto, enquanto as respostas incorretas, seja por não ter conseguido identificar o gosto, seja por tê-lo confundido com outro gosto, não somavam pontos. Para as duas fitas sem gosto também não era atribuida pontuação. Dessa forma, a pontuação máxima do teste foi 16 e utilizou-se o ponto de corte proposto no estudo validado ${ }^{3}$ para classificação de disgeusia (redução do paladar): menor ou igual a 8 pontos. Os idosos foram orientados a não ingerir alimento ou bebida (exceto água), não fumar e não escovar os dentes pelo menos uma hora antes da realização do teste.

Para avaliação do estado nutricional foram aferidas as medidas antropométricas de peso e altura, através de balança mecânica (marca Cauduro $\AA$, com variação de 100g) e antropômetro vertical fixo à balança, respectivamente, estando os individuos descalços e usando roupas leves. Posteriormente, determinou-se o Índice de Massa Corporal (IMC), obtido pela relação entre o peso $(\mathrm{Kg})$ dividido pelo quadrado da altura (m). Utilizaram-se os pontos de corte preconizados pelo Ministério da Saúde do Brasil ${ }^{17}$ e por Lipschitz ${ }^{18}$ para classificação do estado nutricional do idoso: IMC $\leq 22$ : baixo peso; IMC entre 22 e 27: eutrofia ou peso adequado e; IMC $\geq 27$ : sobrepeso.

Aplicou-se um questionário com perguntas fechadas investigando alguns hábitos alimentares: 1) local onde realiza as refeições na maior parte dos dias (casa/restaurante ou lanchonete); 2) costume de adicionar sal na comida depois de pronta (sim/ não); 3) utilização de temperos industrializados ricos em sódio no preparo das refeições (sim/ não/uma vez por semana ou menos); e 4) uso de temperos nas saladas (vinagre/limão/sal/óleo ou azeite de oliva/outros/sem tempero). Inicialmente, as questões referentes ao consumo de sódio (adicionar mais sal na comida depois de pronta; temperar salada com sal ou outros temperos ricos em sódio; utilizar temperos industrializados ricos em sódio para preparar as refeições) foram analisadas separadamente; no entanto, posteriormente, optou-se por agrupar os individuos que responderam positivamente a pelo menos uma dessas questões em uma mesma categoria, denominada "elevado consumo de sódio", para, assim, avaliar o consumo elevado de sódio existente na amostra.

As variáveis contínuas foram expressas como médias \pm desvio-padrão e as variáveis categóricas como frequências relativas e absolutas. Os 
dados foram analisados estatisticamente através dos testes qui-quadrado e ANOVA, por meio do software Epi Info ${ }^{\circledR}$, sendo adotado um nivel de significância de $p<0,05$.

\section{Resultados}

Foram avaliados 62 idosos dos 150 participantes do CELARI com 60 anos ou mais, correspondendo a $41 \%$ dos idosos que aceitaram participar do estudo. A média de idade dos participantes foi 70ะ7,6 anos (variando entre 60 e 85 anos), havendo $84 \%$ do sexo feminino $(n=52)$. A prevalência de disgeusia encontrada foi de 19,4\% ( $n=12)$.

A Tabela 1 apresenta as respostas obtidas para cada uma das fitas oferecidas. Dos quatro gostos testados, o doce encontrou-se mais preservado entre os idosos, visto que 96,8\% identificaram-no corretamente na concentração mais forte e, mesmo na concentração mais fraca, 51,6\% conseguiram reconhecê-lo. Por outro lado, a capacidade para identificar o gosto ácido mostrou-se mais prejudicada, sendo esse reconhecido corretamente na concentração mais fraca por apenas $17,7 \%$ dos individuos e, na concentração mais forte, por $75,8 \%$. Nas concentrações mais fracas para os gostos salgado e amargo, cerca de metade dos participantes referiram não sentir gosto algum (46,7\% e $54,8 \%$, respectivamente). Além disso, tanto o gosto salgado quanto o amargo foram frequentemente confundidos com o gosto ácido nas diferentes concentrações testadas variando de 9,6 a 17\% para o salgado e de 8 a 17,7\% para o amargo.

TABELA 1 - Respostas obtidas pelos idosos para cada tira gustativa ("tastestrip") testada (N = 62)

\begin{tabular}{|c|c|c|c|c|c|c|}
\hline \multirow{2}{*}{$\begin{array}{l}\text { Tiras gustativas } \\
\text { em diferentes } \\
\text { concentrações* }\end{array}$} & \multirow{2}{*}{$\begin{array}{c}\text { Respostas } \\
\text { corretas }^{* *} \\
\mathrm{~N}(\%)\end{array}$} & \multicolumn{5}{|c|}{ Respostas incorretas ${ }^{* *}$} \\
\hline & & Sem gosto & Doce & Ácido & Salgado & Amargo \\
\hline Doce 1 & $60(96,8 \%)$ & 1 & & & & 1 \\
\hline Doce 2 & $54(87,1 \%)$ & 3 & & & & 5 \\
\hline Doce 3 & $47(75,8 \%)$ & 7 & & 3 & 3 & 2 \\
\hline Doce 4 & $32(51,6 \%)$ & 24 & & 3 & 2 & 1 \\
\hline Ácido 1 & $47(75,8 \%)$ & & & & 6 & 9 \\
\hline Ácido 2 & $46(74,2 \%)$ & & & & 8 & 8 \\
\hline Ácido 3 & $34(54,8 \%)$ & 3 & 1 & & 18 & 6 \\
\hline Ácido 4 & $11(17,7 \%)$ & 34 & 5 & & 11 & 1 \\
\hline Salgado 1 & $49(79,0 \%)$ & & & 7 & & 5 \\
\hline Salgado 2 & $44(70,1 \%)$ & 1 & 3 & 11 & & 3 \\
\hline Salgado 3 & $39(62,9 \%)$ & 8 & 1 & 9 & & 5 \\
\hline Salgado 4 & $41(66,1 \%)$ & 29 & 4 & 6 & & 2 \\
\hline Amargo 1 & $46(74,2 \%)$ & 3 & & 11 & 2 & \\
\hline Amargo 2 & $45(72,6 \%)$ & 7 & & 8 & 2 & \\
\hline Amargo 3 & $39(62,9 \%)$ & 14 & & 7 & 2 & \\
\hline Amargo 4 & $18(29,0 \%)$ & 34 & 3 & 5 & 2 & \\
\hline Branco 1 & $58(93.5 \%)$ & & 1 & 2 & & 1 \\
\hline Branco 2 & $51(82,2 \%)$ & & 2 & 5 & 1 & 3 \\
\hline
\end{tabular}

"O número 1 refere-se à concentração mais alta do gosto testado, enquanto 4 refere-se à concentração mais baixa Isacarose 0,$4 ; 0,2 ; 0,1 ; 0,05 \mathrm{~g} / \mathrm{ml}$ (doce); cloreto de sódio 0,25;0,1;0,04;0,016g/ml (salgado); cloridrato de quinino 0,006; 0,0024; 0,0009; 0,0004 g/ml (amargo); e ácido citrico 0,3; 0,165; 0,09; 0,05 g/ml (ácido)]. A ordem apresentada nesta tabela não corresponde à ordem empregada para o teste. Cada conjunto de tiras gustativas ("tastestrips") incluiu duas tiras sem sabor impregnado ("branco").

*A coluna "Respostas corretas" indica o número e a porcentagem de participantes que identificaram corretamente o gosto testado, já a coluna "Respostas incorretas" indicam o número de individuos que erroneamente identificaram cada concentração dos diferentes gostos testados. 
A Tabela 2 apresenta a distribuição dos indivíduos sem e com alteração de paladar de acordo com o estado nutricional e os hábitos alimentares investigados. Detectou-se prevalência de 58\% de sobrepeso ( $n=36)$ e $40,5 \%$ de eutrofia $(n=25)$ na amostra estudada, sendo que apenas um individuo apresentou baixo peso. Os idosos com sobrepeso apresentaram um percentual maior de alteração de paladar quando comparados aos eutróficos, embora não tenha sido encontrada associação significativa entre o estado nutricional e a função gustativa $(p=0,397)$. Os individuos eutróficos fizeram, em média, 10,3 $\pm 2,65$ pontos no teste; os com sobrepeso, $10 \pm 2,84$ pontos; e o único sujeito com baixo peso fez 14 pontos ( $p=0,361$ ) (dados não apresentados nas tabelas).

Aproximadamente $90 \%$ dos idosos $(n=55)$ referiram realizar suas refeições em casa. Não foi encontrada diferença significativa na prevalência de alteração de paladar entre os idosos que realizavam as refeições em casa ou em restaurante (Tabela 2 ).

Verificou-se que aproximadamente 55\% dos participantes $(n=34)$ apresentavam pelo menos um hábito relacionado ao consumo elevado de sódio. A prevalência de alteração de paladar entre individuos com consumo elevado de sódio foi maior do que a verificada entre aqueles sem consumo elevado de sódio, mas sem diferença significativa ( $p=0,278)$ (Tabela 2$)$.

Em relação ao hábito de temperar as saladas, 73\% dos idosos utilizavam azeite de oliva, 60\% vinagre, $52 \%$ sal, $15 \%$ limão, 6,5\% temperos como iogurte e maionese e $5 \%$ shoyo ou temperos prontos ricos em sódio. Os que referiram utilizar limão para temperar a salada tiveram mais facilidade para detectar o gosto ácido do que os que não possuem esse hábito $(2,8 \pm 0,78$ versus $2,1 \pm 1,13$ pontos; $p=0,054)$. Já o uso de vinagre não teve influência sobre o desempenho das pessoas para detectar o gosto ácido, já que ambos obtiveram média de pontos muito semelhante (2,2 $\pm 1,13$ versus $2,2 \pm 1,12 ; p=0,935)$.

Por outro lado, os indivíduos que utilizam sal para temperar a salada ou usam temperos industrializados ricos em sódio para cozinhar apresentaram uma tendência de dificuldade para detectar o gosto salgado quando comparados aos que não possuem esses hábitos $[2,2 \pm 1,02$ versus $2,7 \pm 0,79$ pontos $(p=0,078) ; 2,3 \pm 0,65$ versus $2,5 \pm 1,03(p=0,055)]$.

TABELA 2 - Distribuição dos indivíduos sem e com alteração de paladar, de acordo com o estado nutricional e os hábitos alimentares

\begin{tabular}{|c|c|c|c|c|c|}
\hline \multirow[t]{2}{*}{ Variáveis } & \multicolumn{2}{|c|}{$\begin{array}{l}\text { Sem alteração } \\
\text { de paladar }\end{array}$} & \multicolumn{2}{|c|}{$\begin{array}{l}\text { Com alteração } \\
\text { de paladar }\end{array}$} & \multirow{2}{*}{$\begin{array}{c}\text { Valor } \\
\mathbf{p}^{*}\end{array}$} \\
\hline & $\mathbf{N}$ & $\%$ & $\mathbf{N}$ & $\%$ & \\
\hline \multicolumn{6}{|l|}{ Estado Nutricional ${ }^{* *}$} \\
\hline Eutrofia & 21 & 84 & 4 & 16 & \multirow{2}{*}{0,397} \\
\hline Sobrepeso & 28 & 77,8 & 8 & 22,2 & \\
\hline \multicolumn{6}{|l|}{ Hábitos alimentares } \\
\hline Realiza refeições em casa & 45 & 81,8 & 10 & 18,2 & \multirow{2}{*}{0,409} \\
\hline Realiza refeições em restaurante ou lanchonete & 5 & 71,4 & 2 & 28,6 & \\
\hline Adiciona mais sal na comida depois de pronta & 3 & 75 & 1 & 25 & 0,587 \\
\hline Utiliza temperos prontos para preparar as refeições ${ }^{* * *}$ & 16 & 80 & 4 & 20 & 0,590 \\
\hline Tempera salada com sal & 24 & 75 & 8 & 25 & 0,201 \\
\hline Tempera salada com shoyo ou outros temperos prontos ricos em sódio & 2 & 66,7 & 1 & 33.3 & 0,481 \\
\hline Elevado consumo de sódio ${ }^{\star \star \star \star}$ & 26 & 76,5 & 8 & 23.5 & 0,278 \\
\hline Amostra total $(n=62)$ & 50 & 80,6 & 12 & 19,4 & \\
\hline
\end{tabular}

*valor p de significância < 0,05; teste qui-quadrado

* apenas um individuo apresentou baixo peso e não foi incluido na análise de associação.

*** os individuos que responderam não usar temperos prontos para cozinhar ou utilizá-los uma vez na semana ou menos foram considerados do mesmo grupo.
${ }^{* * * *}$ categoria criada para agrupar os individuos que responderam positivamente a pelo menos uma das questões: adicionar mais sal na comida depois de pronta; temperar salada com sal, shoyo ou outros temperos ricos em sódio; utilizar temperos industrializados ricos em sódio para preparar as refeições. 


\section{Discussão}

O presente estudo contribuiu fornecendo dados de prevalência de disgeusia em uma amostra de idosos. Os poucos estudos existentes ${ }^{10,19,20}$ parecem concordar que os distúrbios sensoriais, principalmente os relacionados ao sistema gustativo, ocorrem com frequência relativamente baixa na população geral e tendem a ganhar maior proporção quando se avalia grupos idosos.

Diferentes resultados são encontrados no que diz respeito aos gostos mais preservados ou afetados com o envelhecimento, dependendo da técnica usada² ou da parte da língua testada.,520 Mas parece haver concordância de que o doce mantém-se mais preservado e, o ácido, mais prejudicado, coincidindo com os achados do presente estudo.

O presente estudo apresentou resultados semelhantes ao encontrados em pesquisa já desenvolvida na área, ${ }^{20}$ em que não foram encontradas associações significativas entre sensibilidade gustativa e diversos fatores como tabagismo, consumo de bebida alcoólica, uso de medicamentos, gênero e estado nutricional, independentemente da concentração testada, em funcionários de uma empresa do ramo de alimentação e em idosos residentes em Instituições de Longa Permanência para Idosos (ILPI). Em outra pesquisa, ${ }^{3}$ verificou-se que os participantes também confundiram os gostos ácido e salgado, achado semelhante ao nosso. Essa confusão pode ser devido à similaridade entre esses gostos, já que ambos podem causar um certo grau de irritação ou "formigamento" na língua, além de serem utilizados em combinação nos alimentos industrializados ricos em tempero. ${ }^{2.3}$

Cabe considerar, ainda, que existe uma variabilidade natural na capacidade dos individuos em perceber e tolerar gostos. Por exemplo, a capacidade de degustar o feniltiocarbamida (PTC), composto presente em alguns alimentos com gosto amargo, é determinada geneticamente, havendo individuos totalmente insensivelao PTC, alguns que necessitam concentrações muito altas para percebê-lo e outros que podem senti-lo em baixissimas concentrações. ${ }^{22}$

A elevada prevalência de sobrepeso encontrada nos idosos avaliados mostra-se compativel com estudos recentes ${ }^{23.24}$ que têm demonstrado o aumento de sobrepeso e de obesidade em diferentes populações idosas. Em estudo que avaliou o estado nutricional de 596 idosos não institucionalizados, ${ }^{25}$ através do IMC, detectou-se alto índice de excesso de peso (48,7\% de acordo com o ponto de corte proposto por Lipchitz e 25.3\% pelo critério da Organização Mundial da Saúde), sobretudo no sexo feminino e na faixa entre 60 e 75 anos. Outro estudo ${ }^{26}$ investigou 117 idosos institucionalizados e encontrou resultados preocupantes: $46,2 \%$ dos idosos apresentavam sobrepeso e, 40,2\%, obesidade grau I.

No presente estudo não foi encontrada associação entre alteração de paladar e estado nutricional, assim como os hábitos alimentares investigados. Entretanto, verificou-se tendência de os individuos com alto consumo de sódio apresentarem maior dificuldade para detectar 0 sabor salgado quando comparados aos que não possuem esse hábito. A respeito disso, sabe-se que as papilas gustativas possuem a capacidade de adaptação tanto para a redução quanto para o aumento do teor de sódio na alimentação e que, se as pessoas não tomam cuidado com relação à quantidade de sal na hora de preparar as refeições, a tendência será exagerar cada dia um pouco mais, sobretudo quando não se utilizam condimentos naturais para realçar o sabor dos alimentos. ${ }^{27}$ Cabe mencionar que a capacidade de detecção do sabor doce, extremamente preservada na amostra estudada, ainda que cerca de metade dos individuos não tenha conseguido identifica-lo na menor concentração, pode ser um dos fatores que predisponha os idosos a um maior consumo de alimentos ricos em açúcares, os quais contribuem para o aumento da prevalência de excesso de peso e de doenças crônicas não transmissiveis nessa população.

Assim, a última versão do Guia Alimentar para a População Brasileira ${ }^{28}$ recomenda utilizar sal, açúcar, óleos e gorduras em pequenas quantidades ao temperar e cozinhar alimentos e criar preparações culinárias, além de limitar o consumo de alimentos processados e evitar os ultraprocessados, por serem ricos nesses ingredientes, 
no intuito de garantir uma alimentação saudável e, com isso, a prevenção de obesidade e de doenças associadas. Ressalta-se a importância de investigar a função gustativa do idoso para identificar estratégias alimentares para a prevenção dos hábitos alimentares não saudáveis nessa população. ${ }^{29}$ Cabe reforçar que o papel do profissional nutricionista é de fundamental importância nessa situação, pois o mesmo pode intervir por meio de uma nutrição adequada, aprimorando o sabor dos alimentos e retardando a progressão de alterações do paladar, contribuindo para um envelhecimento saudável, prazeroso e com qualidade nutricional. ${ }^{30,31}$

Os achados do presente estudo devem ser analisados considerando algumas limitações, sendo uma delas o fato da amostra estudada ter sido composta por idosos ativos, vinculados a um centro de atividade física, além de não terem sido excluidos idosos tabagistas e/ou em uso de medicações com potencial interferência no paladar; assim, pode ser que a prevalência de disgeusia na população idosa geral seja maior e que associações com variáveis nutricionais se tornem mais evidentes, reforçando a necessidade de mais estudos envolvendo amostras maiores e com idosos em diferentes contextos.

\section{Conclusão}

A elevada prevalência de disgeusia encontrada nesta amostra de idosos aponta para a necessidade de novos estudos visando elucidar a magnitude do problema na faixa etária em questão. Embora não tenha sido encontrada associação significativa entre hábitos alimentares investigados, estado nutricional e alteração de paladar, verificou-se um alto percentual de idosos com sobrepeso e com elevado consumo de sódio. Visto que ambos fatores são considerados de risco para o desenvolvimento de inúmeras doenças crônicas não transmissiveis, tais como hipertensão arterial, diabetes e diferentes tipos de câncer, sugere-se que essas variáveis devam ser consideradas em trabalhos futuros, tendo em vista as potenciais repercussões no estado de saúde da população idosa.

\section{Agradecimentos}

Dr. Christian Mueller, da Medical University Vienna (Áustria), por gentilmente fornecer os kits de "taste strips". Eliane e Diná, coordenadoras do CELARI/UFRGS.

\section{Referências}

1. Mistretta CM. Aging Effects on Anatomy and Neurophysiology of Taste and Smell. Gerodontology. 1984:3(2):131-6. https://doi.org/10.1111/j.1741-2358.1984. tbo0364.x

2. Ahne G, Erras A, Hummel T, Kobal G. Assessment of Gustatory Function by Means of Tasting Tablets. Laryngoscope. 2000;110(8):1396-401. https://doi. org/10.1097/00005537-200008000-00033

3. Mueller C, Kallert S, Renner B, Stiassny K, Temmel AF, Hummel T, Kobal G. Quantitative assessment of gustatory function in a clinical context using impregnated "taste strips". Rhinology. 2003;41(1):2-6.

4. Gudziol H, Hummel T. Normative values for the assessment of gustatory function using liquid tastants. Acta Oto-Laryngol. 2007:127(6):658-61. https://doi. org/10.1080/00016480600951491

5.Nordin S, Brämerson A, Bringlöv E, Kobal G, Hummel $\mathrm{T}$, Bende M. Substance and tongue-region specific loss in basic taste-quality identification in elderly adults. Eur Arch Otorhinolaryngol. 2007:264(3):285-9. https://doi. org/10.1007/s00405-006-0169-9

6. Landis BN, Welge-Luessen A, Brämerson A, Bende M, Mueller CA, Nordin S, Hummel T. "Taste Strips" - A rapid, lateralized, gustatory bedside identification test based on impregnated filter papers. J Neurol. 2009; 256(2):2428. https://doi.org/10.1007/s00415-009-0088-y

7. Yen PK. Nutrition and sensory loss. Geriatric Nurs. 2004:25(2):118-9. https://doi.org/10.1016/j.gerinurse.2003.12.011

8. Paula RS, Colares FCJ, Oliveira JN, Nóbrega O. Alterações gustativas no envelhecimento. Rev Kairós. 2008:11(1):217-35

9. Boyce JM, Shone GR. Effects of ageing on smell and taste. Postgrad Med J. 2006;82(966):239-41. https://doi. org/10.1136/pgmj.2005.039453

10. Pribitkin E, Rosenthal MD, Cowart BJ. Prevalence and causes of severe taste loss in a chemosensory clinic population. Ann Otol Rhinol Laryngol. 2003;112(11):971-8. https://doi.org/10.1177/000348940311201110

11. Schiffman SS. Taste and smell losses in normal aging and disease. JAMA. 1997;278(16):1357-62. https://doi. org/10.1001/jama.1997.03550160077042

12. Pavlos P, Vasilios N, Antonia A, Dimitrios K, Georgios K, Georgios A. Evaluation of young smokers and non-smokers with Electrogustometry and Contact Endoscopy. BMC Ear Nose Throat Disord. 2009:9(9). https://doi.org/10.1186/1472-6815-9-9 
13. Kina L, Belotti A, Bruneti RF. Alterações da sensibilidade gustativa no paciente idoso. Atual Geriatr. 1998:3(18):20-2.

14. Cambraia RPB. Aspectos psicobiológicos do comportamento alimentar. Rev Nutr. 2004:17(2):217-25. https://doi.org/10.1590/S1415-52732004000200008

15. Campos MTFS, Monteiro JBR, Ornelas APRC. Fatores que afetam o consumo alimentar e a nutrição do idoso. Rev Nutr. 2000;13(3):157-65. https://doi.org/10.1590/ $\underline{\text { S1415-52732000000300002 }}$

16. Vidal L, Cadena RS, Antúnez L, Giménez A, Varela P, Ares G. Stability of sample configurations from projective mapping: How many consumers are necessary. Food Qual Prefer. 2014:34:79-87. https://doi.org/10.1016/j. foodqual.2013.12.006

17. Brasil. Ministério da Saúde. Orientações para a coleta e análise de dados antropométricos em serviços de saúde [Internet]. Norma Técnica - SISVAN. Brasilia, DF: MS; 2004 [cited 10 abr 2020]. Disponivel em: http://tabnet.datasus.gov.br/cgi-win/SISVAN/CNV/ notas_sisvan.html.

18. Lipschitz, DA. Screening for nutritional status in the elderly. Primary Care. 1994; 21(1): 55-67.

19. Hoffman HJ, Ishii EK, Macturk RH. Age-related changes in the prevalence of smell/taste problems among the United States adult population. Results of the 1994 Disability supplement to the National Health Interview Survey (NHIS). Ann N Y Acad Sci. 1998:855:71622. https://doi.org/10.1111/j.1749-6632.1998.tb10650.x

20. Neumann L, Schauren BC, Adami FS. Sensibilidade gustativa de adultos e idosos. Rev Bras Geriatr Gerontol. 2016;19(5):797-808. https://doi.org/10.1590/1809.98232016019 .150218

21. Strapasson GC, Lopez ACM, Basso T, Santos DF, Mulinari RA, Wille GMFC, et al. Percepção de sabor: uma revisão. Visão Acad. 2011;(12)1:65-73. https://doi. org/10.5380/acd.v12i1.27247

22. Schimidt IL, Oliveira JS, Assis SO, Simões FV, Pratte-Santos R, Freire SCV. Sensibilidade gustativa à feniltiocarbamida. Rev Bras Pesq Saúde. 2018;20(1):148-56. https://doi.org/10.21722/rbps.v20i1.20619

23. Lima LM, Souza RJS, Cunha MRH, Leopoldo AS, Lima-Leopoldo AP. Prevalência de Sobrepeso e Obesidade em Idosas do Centro de Convivência para a Terceira Idade de Vitória/ES. Rev Bras Ciênc Saúde. 2017;21(2):119-126. https://doi.org/10.22478/ufpb. 2317-6032.2017v21n2.22.921

24. Pereira IFS, Spyrides M77HC, Andrade LMB. Estado nutricional de idosos no Brasil: uma abordagem multinivel. Cad Saúde Pública. 2016;32(5): e00178814. https://doi.org/10.1590/0102-311X00178814

25. Silveira E, Kac G, Barbosa L. Prevalência e fatores associados à obesidade em idosos residentes em Pelotas, Rio Grande do Sul, Brasil: classificação da obesidade segundo dois pontos de corte do indice de massa corporal. Cad Saúde Pública. 2009:25(7):1569-77. https://doi.org/10.1590/S0102-311X2009000700015
26. Leite-Cavalcanti C, Rodrigues-Gonçalves MC, Rios-Asciutti LS, Leite-Cavalcanti A. Prevalência de doenças crônicas e estado nutricional em um grupo de idosos brasileiros. Rev Salud Pública. 2009;11(6):865-77. https:// doi.org/10.1590/S0124-00642009000600003

27. Proença RPC, Sousa AA, Veiros MB, Hering B. Qualidade nutricional e sensorial na produção de refeições. Florianópolis: UFSC; 2005.

28. Brasil. Ministério da Saúde. Secretaria de Atenção à Saúde. Departamento de Atenção Básica. Guia alimentar para a população brasileira [Internet]. 2. ed. E 1. reimpr. Brasília: Ministério da Saúde; 2014 lcited 10 abr 2020]. Disponivel em: https://bvsms.saude.gov.br/ bvs/publicacoes/guia_alimentar_populacao_brasileira_2ed.pdf.

29. Sergi G, Bano G, Pizzato S, Veronese N, Manzato E. Taste loss in the elderly: possible implications for dietary habits. Crit Rev Food Sci Nutr. 2017:57(17):3684-9. https://doi.org/10.1080/10408398.2016.1160208

30. Ceolin J, Pinheiro TLF. Sensibilidade gustativa em idosos. Pajar. 2017:5(2):78-84. https://doi. org/10.15448/2357-9641.2017.2.28259

31. Pieroni DCB, Carlins NC, Polato P, Mezzomo TR. Sensibilidade gustativa e estado nutricional de idosas participantes de um Centro Municipal de Atividades para Idosos, Curitiba-PR. O Mundo da Saúde. 2017:41(2):20311. https://doi.org/10.15343/0104-7809.20174102203211

\section{Endereço para correspondência}

Janaina Guimarães Venzke

Universidade Federal do Rio Grande do Sul, Faculdade de Medicina,

Rua Ramiro Barcelos, 2400, $4^{\circ}$ andar, sala 406 90035003

Porto Alegre, RS, Brasil 